\title{
Thermodynamics of atmospheric circulation on hot Jupiters
}

\author{
J. Goodman \\ Princeton University Observatory, Princeton, NJ 08544 \\ jeremy@astro.princeton.edu
}

\begin{abstract}
Atmospheric circulation on tidally-locked exoplanets is driven by the absorption and reradiation of heat from the host star. They are natural heat engines, converting heat into mechanical energy. A steady state is possible only if there is a mechanism to dissipate mechanical energy, or if the redistribution of heat is so effective that the Carnot efficiency is driven to zero. Simulations based on primitive, equivalent-barotropic, or shallow-water equations without explicit provision for dissipation of kinetic energy and for recovery of that energy as heat, violate energy conservation. More seriously perhaps, neglect of physical sources of drag may overestimate wind speeds and rates of advection of heat from the day to the night side.
\end{abstract}

Subject headings: hydrodynamics - waves - binaries: close - planetary systems stars: oscillations, rotation

\section{Introduction}

Showman et al. (2007) have called attention to the divergence in results among attempts to simulate atmospheric ciculation on strongly irradiated jovian exoplanets ("hot Jupiters"). Simulations that allow explicitly for absorption and rejection of heat by the atmosphere, whether by simple Newtonian-cooling schemes or by radiative transfer, generally find prograde equatorial wind speeds $1-5 \mathrm{~km} \mathrm{~s}^{-1}$ (Showman \& Guillot 2002; Burkert et al. 2005; Cooper \& Showman 2005; Langton \& Laughlin 2008; Showman et al. 2008a; Dobbs-Dixon \& Lin 2008). For comparison, the sound speed of nondegenerate molecular hydrogen is approximately $2.4\left(T / 10^{3} \mathrm{~K}\right)^{1 / 2} \mathrm{~km} \mathrm{~s}^{-1}$, so many of these winds are supersonic. Simulations that represent uneven heating indirectly, via prescribed lateral variations in the thickness or depth of the circulating layer, typically have peak winds $<1 \mathrm{~km} \mathrm{~s}^{-1}$ (Cho et al. 2003; Langton \& Laughlin 2007; Cho et al. 2008), and they sometimes predict that the equatorial flow is retrograde. In the simulations of Cho et al. (2003) and Cho et al. (2008), the root-mean-square wind 
speed is put in by hand in the initial conditions. Unlike wind speeds, light curves of these planets have been directly measured, or at least constrained, by infrared observations (Harrington et al. 2006, 2007; Cowan et al. 2007; Knutson et al. 2007). The models generally do not predict these light curves very well: in particular, they sometimes produce a 60-90 ${ }^{\circ}$ prograde shift of the photospheric temperature extrema with respect to the substellar and antistellar points (Cooper \& Showman 2005; Langton \& Laughlin 2007), and they sometimes predict shifts of the wrong sign, as for the cold spot on HD187933b (Showman et al. 2008a).

There may be many reasons for the disagreements among the models and between the

models and the observations. For example, with the exception of those by Burkert et al. (2005) and by Dobbs-Dixon \& Lin (2008), the calculations use approximations based in part on the presumption of subsonic flow, even though the results sometimes violate that presumption. The purpose of the present paper, however, is to emphasize one aspect of the physics that has not been adequately respected by any of the simulations: namely, that atmospheric circulation is a natural heat engine, and that dissipation of mechanical energy must therefore be explicitly addressed. While it may not be easy to decide how dissipation occurs on real planets, it is possible that if a common model for dissipation were adopted, then the calculations might agree better on gross features of the flow such as peak wind speeds and light curves. Without such a common model, convergence to a common result seems unlikely.

\section{Production of mechanical work}

Three of the most recent models of radiatively driven circulation on exoplanets have been based on direct integration of the so-called "primitive equations" adapted from terrestrial meteorology (Showman et al. 2008a; Menou \& Rauscher 2008; Showman et al. 2008b). Compared to previous efforts to model the dynamics of atmospheric circulation, Showman et al. $(2008 \mathrm{~b})$ in particular have significantly improved the model for coupling the flow to the radiation field. Our discussion does not do justice to those improvements, because our concern is with the dynamical part of the model, which has much in common with other efforts, including some of those that employ height-averaged versions of the primitive equations: more detail is given in $\$ 5$.

The primitive equations are

$$
\frac{D \boldsymbol{v}_{\mathrm{h}}}{D t}=-\nabla_{\mathrm{h}} \Phi-f \hat{\boldsymbol{k}} \times \boldsymbol{v}_{\mathrm{h}}
$$




$$
\begin{gathered}
\frac{\partial \Phi}{\partial p}=-\frac{1}{\rho} \\
\boldsymbol{\nabla}_{\mathrm{h}} \cdot \boldsymbol{v}_{\mathrm{h}}+\frac{\partial \omega}{\partial p}=0, \\
\frac{D s}{D t}=\frac{q}{T} .
\end{gathered}
$$

The subscript " $h$ " denotes the two horizontal components of a vector or vectorial operator parallel to isobaric surfaces. The quantity $\Phi=g z$ is the gravitational potential; $\hat{\boldsymbol{k}}$ is the unit normal to the isobars; $f \equiv 2 \boldsymbol{\Omega} \cdot \hat{\boldsymbol{k}}$ is the planetary vorticity, so that $f \hat{\boldsymbol{k}} \times \boldsymbol{v}_{\mathrm{h}}$ is the projection of the Coriolis acceleration onto the isobars; $\rho$ is the mass density; $\omega \equiv D p / D t$ is the vertical "velocity" in pressure coordinates; $s$ and $q$ are the entropy and radiative heating rate per unit mass, respectively; and

$$
\frac{D}{D t}=\frac{\partial}{\partial t}+\boldsymbol{v}_{\mathrm{h}} \cdot \nabla_{\mathrm{h}}+\omega \frac{\partial}{\partial p}
$$

is the time derivative following the flow. Equation (2) is a statement of hydrostatic equilibrium perpendicular to the isobars, so that vertical accelerations have been neglected. This is a good approximation on large horizontal scales, since the vertical pressure scale height is small compared to the radius of the planet $\left(H_{p} \sim 10^{-2} R\right)$, but it precludes explicit representation of small-scale three-dimensional turbulence, which might be an important dissipation mechanism. The continuity equation (3) allows for seepage of material across the isobars. Showman et al. (2008b) evaluate the heating rate $q$ in the entropy equation from the vertical divergence $g \partial F / \partial p$ of the radiative flux, while Showman et al. (2008a) and Menou \& Rauscher (2008) use simpler Newtonian schemes in which $q \propto\left(T-T_{\text {eq }}\right) / \tau$, where $T_{\text {eq }}$ is some prescribed reference temperature, which varies with position, and $\tau$ is a prescribed equilibration time. Equation (44) is often rewritten in terms of the temperature and the specific heat at constant pressure, $c_{p}$; Showman et al. (2008b) rewrite it in terms of potential temperature $\Theta \equiv T\left(p / p_{0}\right)^{(\gamma-1) / \gamma}$ on the assumption that the adiabatic index $\gamma \equiv c_{p} / c_{v}$ is constant. What is important here is that $q$ is interpreted to include radiative heating only; other sources of entropy such as viscous dissipation have been neglected in the simulations that we describe.

One can derive an expression for the rate of production of mechanical energy based on 
the equations above. The first steps are

$$
\begin{aligned}
\frac{D}{D t}\left(\frac{v_{\mathrm{h}}^{2}}{2}\right)=\boldsymbol{v}_{\mathrm{h}} \cdot \frac{D \boldsymbol{v}_{\mathrm{h}}}{D t} & =-\boldsymbol{v}_{\mathrm{h}} \cdot \nabla_{\mathrm{h}} \Phi & & \text { from (1) } \\
& =-\frac{D \Phi}{D t}+\omega \frac{\partial \Phi}{\partial p}+\frac{\partial \Phi}{\partial t} & & \text { using (5) } \\
& =-\frac{D \Phi}{D t}-\frac{\omega}{\rho}+\frac{\partial \Phi}{\partial t} & & \text { using (2) . }
\end{aligned}
$$

Recasting $\omega / \rho=\rho^{-1} D p / D t$ as $D\left(p \rho^{-1}\right) / D t-p D\left(\rho^{-1}\right) / D t$ and rearranging terms results in

$$
\frac{D}{D t}\left(\frac{1}{2} \boldsymbol{v}_{\mathrm{h}}^{2}+\Phi+\frac{p}{\rho}\right)-\frac{\partial \Phi}{\partial t}=p \frac{D}{D t} \frac{1}{\rho} \text {. }
$$

The next step is to integrate eq. (7) over the mass of the atmosphere down to a depth or pressure level sufficiently great that all time derivatives below that level can be neglected, but sufficiently shallow so that the vertical gravity $g \equiv \partial \Phi / \partial z$ can be treated as spatially as well as temporally constant. To do this for the partial time derivative of the potential, which is evaluated at constant pressure rather than constant depth, we use eq. (2) to write the mass element $\mathrm{d} m \equiv \rho \mathrm{d}^{3} \boldsymbol{r}$ as $\mathrm{d} m=g^{-1} \mathrm{~d} p \mathrm{~d} A$, where $\mathrm{d} A$ is an element of area on the isobars 1$]$

$$
\int\left(\frac{\partial \Phi}{\partial t}\right)_{p} \mathrm{~d} m=\int\left(\frac{\partial \Phi}{\partial t}\right)_{p} \frac{\mathrm{d} p \mathrm{~d} A}{g}=\frac{\mathrm{d}}{\mathrm{d} t} \int \Phi \frac{\mathrm{d} p \mathrm{~d} A}{g}=\frac{\mathrm{d}}{\mathrm{d} t} \int \Phi \mathrm{d} m=\int \frac{D \Phi}{D t} \mathrm{~d} m .
$$

The integral of eq. (7) over the mass of the atmosphere is therefore

$$
\frac{\mathrm{d}}{\mathrm{d} t} \int\left(\frac{v_{\mathrm{h}}^{2}}{2}+\frac{p}{\rho}\right) \mathrm{d} m=\int p \frac{D}{D t} \rho^{-1} \mathrm{~d} m .
$$

Using the First Law of Thermodynamics $\mathrm{d} \varepsilon=T \mathrm{~d} s-p \mathrm{~d}\left(\rho^{-1}\right)$, where $\varepsilon$ is the internal energy per unit mass, we may write eq. (9a) in the equivalent form

$$
\frac{\mathrm{d}}{\mathrm{d} t} \int\left(\frac{v_{\mathrm{h}}^{2}}{2}+\varepsilon+\frac{p}{\rho}\right) \mathrm{d} m=\int T \frac{D s}{D t} \mathrm{~d} m .
$$

The integrand on the left side is the Bernoulli constant of the flow, in which $\Phi$ does not appear because the work done against gravity is balanced by the vertical component of the pressure gradient due to the assumption of vertical hydrostatic equilibrium.

\footnotetext{
${ }^{1}$ We have assumed in eq. (8) that $g^{-1} \mathrm{~d} A$ is constant at a given horizontal position on an isobar as it rises or subsides. This would be true in a plane-parallel geometry with strictly constant gravity, since the curvature of the isobars would then be second order in the small ratio of the vertical and horizontal lengthscales. But in spherical geometry, changes in $g$ and $\mathrm{d} A$ can be first order in vertical motions: for example, a spherical isobar of varying radius $r_{p}(t)$, has area $A \propto r_{p}^{2}$ and gravity $g_{p} \propto r_{p}^{-2}$ so that $g_{p}^{-1} \mathrm{~d} A_{p} \propto r_{p}^{4}$. We neglect this complication since we are mainly concerned with the possibility of steady-state circulation, where the isobars would be fixed and $(\partial \Phi / \partial t)_{p}$ would vanish a fortiori.
} 


\section{Frictionless heat engines}

The integrals on the left sides of eqs. (9) are functions of state. Therefore in the absence of secular changes, the time averages of the left sides vanish. But the integrands on the right sides are not time derivatives of functions of state, and so their averages will not vanish automatically. According to equation (4), the entropy of each fluid element changes only by radiative exchange of heat; generation of heat by viscous dissipation has not been allowed for. Insofar as the elements that lose heat are cooler than average (those on the night side), and those that gain it are warmer than average (those on the day side), the righthand side of equation (9b) should be positive. The radiatively driven circulation is a heat engine, continuously producing mechanical energy by tapping the flow of heat from the day to the night side.

Real heat engines, whether artificial or natural, have loads: the mechanical energy that they produce is ultimately returned to heat through an irreversible process. Engineers minimize the dissipation that occurs within the engine itself in order to maximize the power transmitted to an external load. The thermodynamic efficiency of the engine is calculated as the fraction of the heat absorbed from the hot reservoir that is converted to work rather than rejected to the cold reservoir; in this calculation, the heat rejected by the external load is not counted. In self-contained natural heat engines, by contrast, dissipation must be entirely internal. For example, the kinetic energy of convection is dissipated viscously, even at very high Reynolds numbers as in stellar convection zones: turbulent cascades or shocks,

if convection is transonic, bring kinetic energy down to small scales where viscosity converts it to heat. To the extent that a hot Jupiter exchanges energy with its environment purely radiatively, so that winds and tides are negligible in the energy balance, and to the extent that its energy content is not secularly changing, all of the heat absorbed from the star must ultimately be re-radiated. In a sense, therefore, the Carnot efficiency is always zero, but only in a sense that would also apply to any heat engine under steady conditions if the heat dissipated in the load were counted.

Equation (44) allows mass elements to gain or lose heat by radiation, and as eq. (9b) shows, these changes can give rise to a net gain in mechanical energy via $p \mathrm{~d} V$ work. But there are no viscous terms in eqs. (11) and (44) to convert mechanical energy back into heat. Therefore, eqs. (1)-(4) describe a frictionless heat engine. The only way that such an engine could reach steady state would by adjusting itself to zero Carnot efficiency: that is, by arranging for the net $p \mathrm{~d} V$ work to vanish. An example would be convection so efficient as to erase the vertical temperature gradient that drives it. Because the relevant temperature gradient for circulation is longitudinal rather than vertical, it is possible that the Carnot efficiency could be annulled by a $90^{\circ}$ phase shift between the temperature contrast and the radiative heat 
exchange rather than by complete eradication of longitudinal temperature gradients. Something like this seems to be going on at the deeper levels of the simulations of Showman et al. (2008b): see their Figure 3. At the shallower levels, however, the temperatures averaged over the day and night sides are clearly above and below average, respectively, as might be expected from the shorter thermal times there, so a dissipative process is required to explain how steady state is reached at these levels.

Although the dissipation that permits a steady state is not acknowledged in the governing equations (11)-(4), it may be determined implicitly. If the equations are appropriately averaged over height/pressure, they reduce to shallow-water equations [more precisely, equivalent-barotropic equations, Salby (1989)] with a height-integrated "pressure" $\Pi \propto \Sigma^{(2 \gamma-1) / \gamma}$, where $\Sigma$ is the height-integrated density (mass per unit area). It is well known that the shallow-water equations permit weak solutions, i.e. nondifferentiable ones, that contain dissipative discontinuities ("shocks"); The amount of dissipation in the flow as it crosses one of these discontinuities is determined by conservation laws even when the dissipative region is unresolved (see, e.g., Leveque 2002). Thus any algorithm that integrates the equations stably, and that would converge to the correct weak solution (for given initial conditions and source terms) in the limit of infinite resolution, will dissipate if the weak solution does, even if the algorithm doesn't use an explicit dissipative term. Indeed, Showman et al. (2008b) call attention to "hydraulic jumps" in their solutions.

Unfortunately, the dissipation at hydraulic jumps may be mathematically well-defined without being physically correct. That is, the dissipation isn't the same as would be obtained from ideal gas dynamics for the same initial conditions and source function, because eqs. (11)(4) are only an approximation to the ideal-gas equations. In particular, the shallow-water jump conditions conserve mass and momentum but not energy. The energy per unit mass plays a role in the shallow-water approximation that is similar to that played by $(-s)$ in ideal gas dynamics: it is conserved in smooth flow and decreases across shocks (Landau \& Lifshitz 1959; Leveque 2002). Inasmuch as eqs. (1)-(41) reduce to shallow-water equations when the horizontal velocities are constant with height, they probably destroy energy at hydraulic jumps. Certainly there is nothing in the equations to convert the dissipated mechanical energy to heat.

\section{Other work}

Menou \& Rauscher (2008) adopt equations equivalent to (11)-(41), but they add dissipative terms. Some of these are diffusive terms for the temperature, vorticity, and velocitydivergence fields. The only terms that would appear to dissipate kinetic energy are terms 
that damp the relative vorticity and velocity divergence; in our notation, these are of the forms

$$
\begin{gathered}
\frac{D}{D t}\left(\boldsymbol{\nabla}_{\mathrm{h}} \times \boldsymbol{v}_{\mathrm{h}}\right)=\ldots-\frac{\boldsymbol{\nabla}_{\mathrm{h}} \times \boldsymbol{v}_{\mathrm{h}}}{\tau_{\text {fric }}} \\
\frac{D}{D t}\left(\boldsymbol{\nabla}_{\mathrm{h}} \cdot \boldsymbol{v}_{\mathrm{h}}\right)=\ldots-\frac{\boldsymbol{\nabla}_{\mathrm{h}} \cdot \boldsymbol{v}_{\mathrm{h}}}{\tau_{\text {fric }}} .
\end{gathered}
$$

For the purpose of testing their codes against previous calculations made for the Earth's atmosphere, they take the friction time $\tau_{\text {fric }}=2 \pi \Omega^{-1}$, but when they apply the model to gaseous exoplanets, they set $\tau_{\text {fric }}=\infty$, perhaps because it is understood that these terms are intended to represent turbulent drag against the Earth's solid surface. Nevertheless, their calculations saturate with subsonic wind speeds, whereas the peak speeds in Showman et al. (2008b), and also in most of the previous calculations by Showman and his collaborators, are supersonic.

Showman \& Guillot (2002) integrate the primitive equations in three dimensions. They do not include explicit dissipative terms, but they do remark upon a downward energy flux at the bottom of their grid, towards the planetary interior. It is not clear whether this is enough of an energy sink by itself to explain the quasi-steady circulation in the atmosphere.

Cho et al. (2003) and Cho et al. (2008) solve two-dimensional shallow-water equationsmore precisely, equivalent barotropic equations. There is no heat engine in these models: thermal "forcing" is represented by a prescribed variation in the effective depth of the circulating layer, but in such a way that no net work is done on the fluid in the time average. For example, the governing equations of Cho et al. (2003) for the horizontal velocity and effective layer thickness, $h$, are (with some minor changes in notation)

$$
\begin{gathered}
\left(\frac{\partial}{\partial t}+\boldsymbol{v}_{\mathrm{h}} \cdot \nabla_{\mathrm{h}}\right) \boldsymbol{v}_{\mathrm{h}}=-g \boldsymbol{\nabla}_{\mathrm{h}}\left(h-h_{a}\right)+f \hat{\boldsymbol{k}} \times \boldsymbol{v}_{\mathrm{h}}, \\
\left(\frac{\partial}{\partial t}+\boldsymbol{v}_{\mathrm{h}} \cdot \boldsymbol{\nabla}_{\mathrm{h}}\right) h=-\mathcal{K} h \boldsymbol{\nabla}_{\mathrm{h}} \cdot \boldsymbol{v}_{\mathrm{h}}-\frac{h-h_{d}}{\tau_{d}} .
\end{gathered}
$$

Here $h_{a}$ and $h_{d}$ are prescribed functions intended to represent the forcing, and $\tau_{d}$ is a constant. Integrating the righthand side of eq. (CMHS.1) around a closed streamline yields zero because the accelerations parallel to the flow are gradients of scalars. Thus, a steady-solution of these equations without dissipative terms could be compatible with energy conservation.2 In fact,

\footnotetext{
${ }^{2}$ This argument does not apply to (1) because streamlines may cross isobaric surfaces. Thus if $A B B^{\prime} A^{\prime}$ is a hypothetical "rectangular" streamline such that the legs $A B$ and $B^{\prime} A^{\prime}$ lie entirely on neighboring isobars $p$ and $p^{\prime}$, then the line integral of the three-dimensional gradient $\nabla \Phi$ does vanish, but the contributions of the vertical legs $B B^{\prime}$ and $A^{\prime} A$ are offset by the pressure acceleration due to hydrostatic equilibrium [eq. (2)], and these contributions do not cancel unless $\rho_{B B^{\prime}}=\rho_{A^{\prime} A}$.
} 
a steady solution with $\boldsymbol{v}_{\mathrm{h}}=0$ and $h=h_{a}$ clearly exists if $h_{d}=h_{a}$. On the other hand, the equations do not conserve the mass in the layer unless $\tau_{d} \rightarrow \infty$, in which case (CMHS.2) can be recast as $\partial \Sigma / \partial t+\nabla_{\mathrm{h}} \cdot\left(\Sigma \boldsymbol{v}_{\mathrm{h}}\right)=0$ for areal mass density $\Sigma \propto h^{1 / \mathcal{K}}$.

Langton \& Laughlin (2007) integrate the shallow-water equations using a spectral method. They do not display any explicit dissipative term in the momentum equations, but they mention the use of a hyperviscosity. Langton \& Laughlin (2008) adopt somewhat different, but also two-dimensional, equations with a radiative forcing that appears to yield a nonconservative force in the momentum equation [the term $R T \nabla_{\mathrm{h}} \ln \rho$ in their eq. (9)], so they may have a heat engine. Their only explicit mechanical dissipation is again a hyperviscosity, $-B \nabla^{4} \boldsymbol{v}_{\mathrm{h}}$, but the mechanical energy lost via this term is apparently not added as heat to the evolutionary equation for the temperature.

Not all of the work in this subject has been carried out with the primitive, shallow-water, or equivalent-barotropic approximations. Burkert et al. (2005) and Dobbs-Dixon \& Lin (2008) use the full ideal-gas equations, including radiative forcing terms but without the approximation of vertical hydrostatic equilibrium. The former work is limited to two dimensions (radius and longitude), while the latter is fully three dimensional. It is not clear from their abbreviated descriptions whether their schemes conserve total energy, but if they do, then these schemes should be correctly converting the mechanical energy of the circulation into heat. Even so, by neglecting explicit dissipation, they may overestimate the flow speed. Dobbs-Dixon \& Lin (2008) remark upon flow speeds up to Mach 2.7 and dissipation in shocks.

\section{Discussion}

Analogies between atmospheric circulation and heat engines have been made before. In the atmospheres of planets such as the Earth whose rotational periods are short compared both to their orbital periods and to the thermal times in their atmospheres 3 , the primary temperature gradient is latitudinal, and the atmospheric heat engine operates between the equator and the poles, via the Hadley, mid-latitude, and polar cells. As discussed by Lorenz et al. (2001, and references therein), the latitudinal circulation on Earth, and perhaps also on Mars and Titan, adjusts itself approximately so as to maximize the rate of entropy production, defined as $\dot{S} \equiv \dot{Q}_{\text {adv }}\left(T_{\text {pole }}^{-1}-T_{\text {equator }}^{-1}\right)$, where $\dot{Q}_{\text {adv }}$ is the rate of poleward heat transport by advection. Here $\dot{S}$ does not represent a secular increase in the entropy of

\footnotetext{
${ }^{3}$ The temperature of the terrestrial atmosphere varies rather little over the diurnal cycle except at low altitude where the air easily exchanges heat with the ground.
} 
the atmosphere itself, since the heat and entropy brought to the poles is ultimately radiated to space, but rather a (small) increase in the entropy of the universe. Maximizing $\dot{S}$ is not equivalent to maximizing Carnot efficiency $\left(\eta_{\mathrm{C}}\right)$, but neither is it compatible with $\eta_{\mathrm{C}}=0$. The entropy production defined this way would vanish for $\dot{Q}_{\mathrm{adv}}=0$, which would lead to the largest difference between equatorial and polar temperatures and hence to the largest possible $\eta_{\mathrm{C}}$; but it would also vanish if the heat transport were so large that $T_{\text {pole }}=T_{\text {equator }}$, when $\eta_{\mathrm{C}} \rightarrow 0$. In the actual state of approximately maximal $\dot{S}$, the mechanical energy of the circulation is continuously produced by the heat engine but dissipated by turbulent friction with the solid surface. Since the transported heat is ultimately radiated, a quantity related to $\dot{S}$ that could be measured in those simulations that include radiative transport might be

$$
\iint_{\text {photosph. }} T_{\mathrm{ph}}^{-1} \boldsymbol{F}_{\mathrm{rad}} \cdot \mathrm{d}^{2} \boldsymbol{A}
$$

where $\boldsymbol{F}_{\text {rad }}$ is the difference between the outward planetary flux and the inward stellar flux, and $T_{\mathrm{ph}}$ is the gas temperature at the photosphere. Since the optical and infrared photospheres do not coincide, however, a better definition might be

$$
\dot{S}_{\mathrm{LL}}=\iiint_{\text {atmos. }} \boldsymbol{\nabla} \cdot\left(\frac{\boldsymbol{F}_{\mathrm{rad}}}{T}\right) \mathrm{d}^{3} \boldsymbol{r},
$$

where the subscript indicates that this is entropy production in the sense of Lorenz et al. (2001). Normalized perhaps by the total insolation and by a mean photospheric temperature, the quantity (10) might be used to assess the degree to which the redistribution of heat is maximal. It is not obvious a priori why $\dot{S}_{\mathrm{LL}}$ should be maximal, however.

There are at least two distinct though related ways in which neglect of explicit dissipation could lead to erroneous results:

1. If mechanical energy is dissipated by numerical effects that do not convert it to heat, then energy is lost. The seriousness of this error for predictions of infrared light curves, etc., depends upon the fraction of the advected energy that is carried in kinetic form rather than as enthalpy. The latter could be measured by integrating the two contributions to the energy flux over the meridional plane at the dawn and dusk terminators, for example.

2. Even when total energy is conserved or its loss is unimportant, wind speeds and rates of redistribution of heat may be overestimated when physical mechanisms of drag on the flow are left out. 
Terrestrial circulation is highly subsonic, so modelers presume that errors of the first type are not serious. This is not so obvious for the models of hot Jupiters. Since the simulators do not report the balance between the kinetic and enthalpic energy fluxes, consider the hypothesis that the wind speed $U$ at each (sufficiently deep) pressure level adjusts itself so that the advection time $\tau_{\text {adv }} \equiv R / U$ is proportional to the thermal time at that depth,

$$
\tau_{\text {th }} \equiv \frac{p}{\sigma T^{4}} \frac{\kappa \rho H_{p}^{2}}{c}=\frac{\kappa P^{2} H_{p}}{g \sigma T^{4}},
$$

where $H_{p} \approx k_{\mathrm{B}} T / 2 m_{p} g$ is the pressure scale height. In an isothermal atmosphere, this hypothesis predicts $U \propto \kappa^{-1} p^{-2}$. The horizontal enthalpy flux would then scale with depth as $(\kappa p)^{-1}$, and the kinetic flux as $\kappa^{-3} p^{-5}$. Since the opacity generally increases with pressure, the depth-integrated heat transport would be dominated by low pressures near the photosphere, where the kinetic component is important inasmuch as the speeds are transonic according to the simulations.

On the other hand, if the simulations have overestimated wind speeds, then the kinetic contribution to the energy flux may turn out to be minor after all. This brings us to point 2 above: What physical mechanisms of dissipation and drag may operate in real exoplanetary atmospheres that have not been included in the simulations? Unlike terrestrial planets, hot Jupiters presumably have no solid surface to provide boundary-layer drag; a dissipative mechanism that is distributed through the atmosphere is required. Two important possibilities are shocks and shear-driven turbulence (perhaps also waves). Shocks would occur in steady flow only if it is supersonic. They might do little to mix the atmosphere and to resist chemical fractionation. If shocks dominate, this would be good for simulations, which ought to be able to capture them, as in the simulations of Burkert et al. (2005) and Dobbs-Dixon \& Lin (2008). If turbulence dominates, however, then the simulations may require a subgrid model, because a necessary characteristic of the turbulence is that it extend to unresolvably small scales where viscosity is effective. The subgrid model should be based on an understanding of the instabilities that promote the turbulence and how they saturate, unless an empirical model can be calibrated against the well-resolved planetary atmospheres of the solar system.

The principal obstacle to the development of three-dimensional turbulence and a cascade to small scales is the stable stratification. The kinetic energy available in the vertical wind shear must be greater than the potential energy required to mix the atmosphere. The ratio of these energies is quantified by the Richardson number $R i \equiv N^{2} /(\partial U / \partial r)^{2}$, where $N$ is the Brunt-Väisälä frequency and $U$ is the horizontal velocity. A requirement for $\mathrm{KH}$ instability in vertical planes is that $R i<1 / 4$ somewhere in the flow, at least under adiabatic and inviscid conditions (Drazin \& Reid 1981). It would be helpful if numerical researchers would 
report the values of $R i$ achieved in their simulations. For an isothermal atmosphere with density and pressure scale height $H_{p}=c_{\mathrm{s}}^{2} / \gamma g$, the condition $R i<\frac{1}{4}$ is equivalent to

$$
\left|\frac{\partial U}{\partial r}\right|>\frac{2 \sqrt{\gamma-1}}{\gamma} \frac{c_{\mathrm{s}}}{H_{p}}
$$

so with $\gamma \approx 7 / 5$ as for nondegenerate molecular hydrogen, the flow must be transonic or else change on a scale smaller than the scale height in order that $R i<1 / 4$. But it is possible that instability may occur at larger Richardson number in the presence of radiative diffusion. Other instabilities that may be relevant are the baroclinic instability (Pedloskv 1987; Vallis 2006), the Goldreich-Schubert-Fricke instability (Goldreich \& Schubert 1967; Fricke 1968), and perhaps even the magnetorotational instability (Balbus \& Hawley 1991) if the shear extends to such a depth that the atmosphere becomes substantially conducting, and if the shear has the right sign. It is beyond the scope of this note to assess the importance of these instabilities for controlling the speed of the circulation. Nevertheless, we will discuss the GSF instability briefly because it is illustrative and must surely occur at some level.

The GSF instability is enabled by thermal diffusion in baroclinic atmospheres of negligible viscosity that do not rotate on cylinders, $\partial \Omega / \partial z \neq 0$, even if the atmosphere is dynamically stable according to Hølland's Criterion (Tassoul 1978), i.e. baroclinically stable. The instability is axisymmetric, and the maximum growth rate is expressed in terms of the cylindrical radius $\varpi$ and angular momentum per unit mass $j=\varpi^{2} \Omega$ by

$$
\operatorname{Im}(\omega)_{\max }=\left[\frac{1}{2 \varpi^{3}}\left(\left|\nabla j^{2}\right|-\frac{\partial j^{2}}{\partial \varpi}\right)\right]^{1 / 2}
$$

but this is approached only at wavelengths $\lambda \lesssim 2 \pi(\chi / N)^{1 / 2}$, where $\chi=16 \sigma T^{3} / 3 \kappa \rho^{2} c_{v} \sim$ $(c / \kappa \rho)\left(p_{\mathrm{rad}} / p\right)$ is the thermal diffusivity, so that radiative diffusion undercuts the restoring force of buoyancy. Thus $\lambda \lesssim 1 \mathrm{~km}(p / \text { bar })^{-1}\left(\kappa / \mathrm{cm}^{2} \mathrm{~g}^{-1}\right)^{-1 / 2}\left(T / 10^{3} \mathrm{~K}\right)^{2}$. Such a wavelength would not be resolved by global simulations. One might expect the GSF instabilities to saturate nonlinearly at displacements $\xi \sim \lambda$ due to nonaxisymmetric Kelvin-Helmholtz instabilities acting on the rising and falling "fingers," which carry oppositely signed eulerian angular-velocity perturbations. However, Goldreich \& Schubert (1967) themselves took the point of view that the saturation occurs with displacements comparable to the pressure scale height, and therefore provides a turbulent viscosity $\sim \Omega H_{p}^{2} / 2 \pi$. This is something best studied by local rather than global simulations, and then represented in the latter by a subgrid model. Korycansky (1991) simulated the nonlinear outcome of the GSF instability in the special case where $\partial j / \partial z=0$ and $N^{2}>-\varpi^{-3} \partial j^{2} / \partial \varpi>0$. His results support Goldreich \& Schubert (1967)'s view of the saturation, but his two-dimensional simulations could not have represented the nonaxisymmetric Kelvin-Helmholtz instabilities most likely 
to limit GSF modes. Arlt \& Urpin (2004) simulated the case $\partial j^{2} / \partial \varpi>0$ and $\partial j^{2} / \partial z<0$ in three dimensions, using ZEUS3D, and concluded that mixing was efficient, but they adopted a barotropic equation of state so that their unperturbed state had to be out of equilib-

rium. Menou et al. (2004) added magnetic effects to the GSF analysis, but only in the linear regime.

While the growth rate of instabilities that rely on thermal diffusion should decrease rapidly with increasing pressure, the rate at which radiative transfer tends to restore the stratification also decreases, so that the outcome for the profiles of entropy and angular velocity is unclear. If any of these instabilities is effective at redistributing angular momentum, then the radiatively driven circulation may go deeper than present simulations suggest, and the time required for the rotation profile to reach steady state may be very long.

We thank Adam Burrows, Kristen Menou, Jonathan Mitchell, Geoffrey Vallis, and the Peyton-Hall astro-ph coffee klatsch for helpful discussions. This work was supported in part by the National Science foundation under grant AST-0707373.

\section{REFERENCES}

Arlt, R. \& Urpin, V. 2004, A\&A, 426, 755

Balbus, S. \& Hawley, J. 1991, ApJ, 376, 214

Burkert, A., Lin, D. N. C., Bodenheimer, P. H., Jones, C. A., \& Yorke, H. W. 2005, ApJ, 618,512

Cho, J. Y.-K., Menou, K., Hansen, B. M. S., \& Seager, S. 2003, ApJ, 587, L117

-. 2008, ApJ, 675, 817

Cooper, C. S. \& Showman, A. P. 2005, ApJ, 629, L45

Cowan, N. B., Agol, E., \& Charbonneau, D. 2007, MNRAS, 379, 641

Dobbs-Dixon, I. \& Lin, D. N. C. 2008, ApJ, 673, 513

Drazin, P. G. \& Reid, W. H. 1981, Hydrodynamic Stability (Cambridge: Cambridge University Press)

Fricke, K. 1968, Zeitschrift für Astrophysik, 317 
Goldreich, P. \& Schubert, G. 1967, ApJ, 150, 571

Harrington, J., Hansen, B. M., Luszcz, S. H., Seager, S., Deming, D., Menou, K., Cho, J. Y.-K., \& Richardson, L. J. 2006, Science, 314, 623

Harrington, J., Luszcz, S., Seager, S., Deming, D., \& Richardson, L. J. 2007, Nature, 447, 691

Knutson, H. A., Charbonneau, D., Allen, L. E., Fortney, J. J., Agol, E., Cowan, N. B., Showman, A. P., Cooper, C. S., \& Megeath, S. T. 2007, Nature, 447, 183

Korycansky, D. G. 1991, ApJ, 381, 515

Landau, L. D. \& Lifshitz, E. M. 1959, Fluid Mechanics (Pergamon Press)

Langton, J. \& Laughlin, G. 2007, ApJ, 657, L113

-. 2008, ApJ, 674, 1106

Leveque, R. J. 2002, Finite Volume Methods for Hyperbolic Problems (Cambridge)

Lorenz, R. D., Lunine, J. I., Withers, P. G., \& McKay, C. P. 2001, Geophys. Res. Lett., 28, 415

Menou, K., Balbus, S. A., \& Spruit, H. C. 2004, ApJ, 607, 564

Menou, K. \& Rauscher, E. 2008, ArXiv e-prints, 809

Pedlosky, J. 1987, Geophysical Fluid Dynamics, 2nd edn. (Springer-Verlag)

Salby, M. L. 1989, Tellus, 41A, 46

Showman, A. P., Cooper, C. S., Fortney, J. J., \& Marley, M. S. 2008a, ApJ, 682, 559

Showman, A. P., Fortney, J. J., Lian, Y., Marley, M. S., Freedman, R. S., Knutson, H. A., \& Charbonneau, D. 2008b, ArXiv e-prints, 809

Showman, A. P. \& Guillot, T. 2002, A\&A, 385, 166

Showman, A. P., Menou, K., \& Y-K. Cho, J. 2007, ArXiv e-prints, 710

Tassoul, J.-L. 1978, Theory of Rotating Stars (Princeton University Press)

Vallis, G. K. 2006, Atmospheric and Oceanic Fluid Dynamics (Cambridge: Cambridge University Press) 
This preprint was prepared with the AAS IATEX macros v5.2. 